\title{
FAULT-TOLERANT CONTROL BASED ON ITERATIVE LEARNING OBSERVER: A DESCRIPTOR APPROACH
}

\author{
ShiLei Zhao,* TieJun Zhang,, and YuPeng Liu*
}

\begin{abstract}
Based on the iterative learning (IL) strategy and unknown input observer (UIO), the problem of actuator fault estimation and fault-tolerant control design for linear systems is studied in this paper. The aim of employing the UIO is to estimate system states accurately for feedback control with the effects of disturbances. The IL update law is adopted to learn the fault by using the previous fault information and the UIO output estimation error. The IL update law which can be referred to time-delay control, the feedback input error, the system state and the state estimation error is organized into a kind of time-delay descriptor system, and the stability of this descriptor system is easily solved in terms of linear matrix inequalities, Lyapunov-Krasovskii stability theory and $\mathrm{H} \infty$ technique. Finally, a numerical example has been studied to illustrate the efficiency of the proposed approach.
\end{abstract}

\section{Key Words}

Iterative learning, Fault-tolerant control, descriptor time delay system, unknown input observer

\section{Introduction}

Many practical control systems are vulnerable to possible malfunctions that may cause performance degradation and even instability of the system. To increase the safety and reliability of dynamic systems, the issues of fault diagnosis and fault tolerant control (FTC) have become an attractive topic, and these issues are widely investigated [1]-[7]. Among these works, the observer is a common approach that has been widely used. The main idea of this strategy is to take the processed errors between the system output and the observer output as the residual to detect, isolate and estimate faults. Fruitful results can be found in several papers [8]-[11].

Recently, there are some studies in the area of fault detection and identification (FDI) based on iterative learning observer (ILO). ILO is an observer design methodology

* School of Software, Harbin University of Science and Technology, Harbin, People's Republic of China; e-mail: zhaosl1210@ hotmail.com, tiejun.zhang@hrbust.edu.cn, flyeagle99@126.com Recommended by Dr. Francesco Pierri

(DOI: 10.2316/Journal.206.2017.2.206-4845) using iterative learning (IL) methods for fault detection and fault estimation. The essence is the use of previous information combined into the ILO for identifying online time-varying faults. In [12], a novel updating mechanism, known as ILO, is first proposed by using the previous information to "learn" the fault and the disturbance simultaneously. However, the approach in [12] cannot estimate the fault exclusively. Another work related to ILO is Jia's [13]. In [13], the ILO design subject to loss of actuator effectiveness is presented for linear parameters varying (LPV) systems. Because of no chattering induced, the ILO is sensitive to incipient faults. However, the method proposed by [14] does not involve FTC problems. From the nonlinear robust control viewpoint, robust FTC based on the IL observer is proposed in studies [11], [15], these two works introduce fault estimation methods by using ILO, and the fault-tolerant controller is designed to guarantee the stability of nonlinear systems. But both works do not consider the effects of the disturbance.

In this work, we propose an approach to detect, estimate and compensate the actuator fault, in which there are no subsystems needed to detect and isolate faults. The design is based on the IL law and unknown input observers (UIOs) which are used to monitor system dynamic variations caused by faults. Once a fault is detected, the state estimation error would excite the IL law to estimate the fault online which is employed to offset the effect of the fault on system dynamics such that the system can be maintained stable. The combination of the fault estimation error, the system state, the system state estimation error and the difference between the nominal input and the FTC input leads to a time-delay descriptor system and the convergence of the descriptor system is proved by using Lyapunov-Krasovskii stability theory and $\mathrm{H} \infty$ technique.

The advantages of the proposed method can be summarized as follows: (1) The different errors are organized into a kind of time-delay descriptor system, therefore, the problem of fault estimation and FTC is solved simultaneously; (2) The introduction of the descriptor redundancy property can avoid crossed terms in linear matrix inequality (LMI) and then decrease the number of LMI conditions and consequently relax the conservatism; (3) IL update 
law is referred to time-delay control which can adopt the Lyapunov-Krasovskii theorem to prove the stability.

This paper is organized as follows. In the following section, the system under study and the IL law are presented, and some assumptions that UIO needs have been given. Then, the actuator fault estimation design based on UIO and the steady conditions based on LMI have been given. In Section 3, a fault-tolerant feedback control law design for linear systems affected by time-varying actuator faults is given. In Section 4, a numerical example for constant faults and time-varying faults is considered to illustrate the applicability and effectiveness of the proposed approaches.

Notations: The following notation will be adopted throughout this paper: $A>0(A<0)$ denotes that $A$ is positive(negative) definite; $*$ means symmetric term, $I_{n}$ is an identity matrix of size $n ; 0_{m}$ is an $m$ row and $m$ column square matrix; $\operatorname{sym}(A)$ denotes $A+A^{T}$ and $\operatorname{diag}\{\cdots\}$ stands for a block-diagonal matrix.

\section{System Description and Design of the Iterative Learning Observers}

Consider the following linear time-invariant faulty system without disturbances:

$$
\begin{aligned}
& \dot{x}(t)=A x(t)+B u(t)+F f(t) \\
& y(t)=C x(t)
\end{aligned}
$$

where $x(t) \in R^{n}, u(t) \in R^{m}, y(t) \in R^{p}$ and $f(t) \in R^{r}$ are the system state vector, the control input vector, the measurement output vector and the unknown fault vector, respectively. $A, B, F, C$ are known constant matrices with appropriate dimensions.

To estimate the system state by using the UIO, the following assumptions are made.

Assumption 1. The matrix $C$ is full-row rank, $\operatorname{rank}(C)=p$.

Assumption 2. A necessary condition for the existence of a stable observer for the system described in (1) is $\operatorname{rank}(C F)=r$.

Throughout the paper, Assumptions 1 and 2 are assumed to be true, that is, the dimensions of the system unknown fault are less than the system output.

For system (1), a kind of UIO is established into the following form:

$$
\begin{aligned}
& \dot{z}(t)=N z(t)+G u(t)+T \hat{f}(t)+L_{1} y(t) \\
& \hat{x}(t)=z(t)+H_{2} y(t) \\
& \hat{f}(t)=\hat{f}(t-h)+K_{1} e_{y}(t)
\end{aligned}
$$

where $\hat{x}(t)$ and $e_{y}(t)$ are the estimated system state and the output estimation error with dimensions of $n$ and $p$, respectively. Variable $\hat{f}(t)$ represents the estimate of the fault updated by its previous information at the sampling time $t-h$ and the updating interval $h$.

Remark 1. It should be noticed that the ILO is updated through the previous information and the output estimation error, the meaning of "learning" is the use of the previous information at sampling time $t-h$. To guarantee the estimation accuracy, the sampling interval $h$ should be adjusted according to [13].

To ensure the accurate estimate, we first give state estimation error $e_{x}(t)$, output estimation error $e_{y}(t)$, and fault estimation error $e_{f}(t)$ represented by:

$$
\begin{aligned}
& e_{x}(t)=x(t)-\hat{x}(t) \\
& e_{y}(t)=y(t)-\hat{y}(t) \\
& e_{f}(t)=f(t)-\hat{f}(t)
\end{aligned}
$$

Then, the state estimation error can be written as:

$$
e_{x}(t)=x(t)-z(t)-H_{2} C x(t)=\left(I-H_{2} C\right) x(t)-z(t)
$$

Assuming that there exists a matrix $H_{1} \in R^{n \times n}$ such that $H_{1}=I-H_{2} C$, by taking into account (2) and (3), the dynamics of the state estimation error is given by:

$$
\dot{e}_{x}(t)=H_{1} \dot{x}(t)-\dot{z}(t)
$$

Substituting (1) and (2) into (4), we have the dynamics of the state estimation error in the form of

$$
\begin{aligned}
\dot{e}_{x}(t)= & N e_{x}(t)+\left(H_{1} A-N H_{1}-L_{1} C\right) x(t) \\
& +\left(H_{1} B-G\right) u(t)+T e_{f}(t)+\left(T-H_{1} F\right) f(t)
\end{aligned}
$$

If the following relations hold true:

$$
\begin{aligned}
& H_{1} A-N H_{1}-L_{1} C=0 \\
& G=H_{1} B \\
& T=H_{1} F
\end{aligned}
$$

we can obtain the parameters of observer (2) which are shown in the following:

$$
\begin{aligned}
& N=H_{1} A-K C \\
& K=L_{1}-N H_{2} \\
& L_{1}=K+N H_{2}
\end{aligned}
$$

From (2), (3) and (6), the dynamics of the state estimation error and the fault estimation error can be described by:

$$
\begin{aligned}
& \dot{e}_{x}(t)=N e(t)+T e_{f}(t) \\
& e_{f}(t)=e_{f}(t-h)-K_{1} C e_{x}(t)+f(t)-f(t-h)
\end{aligned}
$$


Assume that the variation of $f(t)$ is bounded with a known bound:

$$
\|f(t)-f(t-h)\| \leq k_{f}
$$

where $h$ is the sampling interval in a sampled-data system.

Then, by using the so-called virtual dynamic [16], (8) can be rewritten as a descriptor system of the form:

$$
E \dot{e}(t)=\left(A_{0}-\tilde{K} \tilde{C}\right) e(t)+A_{d} e(t-h)+R w(t)
$$

where $E=\operatorname{diag}\left\{I_{n}, 0_{r}\right\}, e(t)=\left[e_{x}^{T}(t) \quad e_{f}^{T}(t)\right]^{T}, w(t)=k_{f}$, $A_{d}=\left[\begin{array}{ll}0 & 0 \\ 0 & I\end{array}\right], R=\left[\begin{array}{l}0 \\ I\end{array}\right], \tilde{K}=\left[\begin{array}{l}K \\ K_{1}\end{array}\right], A_{0}=\left[\begin{array}{cc}H_{1} A & T \\ 0 & -I\end{array}\right]$, $\tilde{C}=\left[\begin{array}{c}C^{T} \\ 0\end{array}\right]^{T}$.

Remark 2. To gain an understanding of this form, the idea of the ILO input update law presented in (2) can be referred to time-delay control [17] or to IL update law [12], [13], [18]. In this paper, we shall refer to it as timedelay control. So, we can use the Lyapunov-Krasovskii stability theory to prove the stability of this system.

The stability and convergence conditions are given in [16], [19], [20].

Theorem 1. System (10) describing the state estimation error $e_{x}(t)$ and the fault estimation error $e_{f}(t)$ are stable and the gain from the perturbation-like term $w(t)$ to the state estimation error and the fault estimation error are bounded by $\gamma>0$. If there exist some matrices $P_{1}, P_{2}>0, P_{3}>0$ and $M>0$, matrix $Y$ with appropriate dimensions such that the following matrix inequalities (11) hold:

$$
\begin{aligned}
& {\left[\begin{array}{ccc}
\Omega & Y & \Psi \\
* & -P_{3} / h & 0 \\
* & * & -M / h
\end{array}\right] \leq 0} \\
& {\left[\begin{array}{cc}
M & P_{1} \\
P_{1} & P_{3}
\end{array}\right] \leq 0}
\end{aligned}
$$

where the $\Omega$ and $\Psi$ is shown in the following:

$$
\begin{aligned}
\Omega= & W_{p}^{T} Q W_{p}-\gamma^{2} W_{w}^{T} W_{w}+W_{p}^{T} P_{2} W_{p}-W_{p h}^{T} P_{2} W_{p h} \\
& +\operatorname{sym}\left(W_{p}^{T}\left(P_{1} A_{0}-X \tilde{C}\right) W_{p}+W_{p}^{T} P_{1} A_{d} W_{p h}\right. \\
& \left.+W_{p}^{T} P_{1} R W_{w}+Y E W_{p}-Y E W_{p h}\right) \\
\Psi= & {\left[\begin{array}{lll}
\left(P_{1} A_{0}-X \tilde{C}\right) & P_{1} A_{d} & P_{1} R
\end{array}\right]^{T} } \\
W_{p}= & {\left[\begin{array}{lll}
I_{n+r} & 0_{n+r} & 0_{(n+r) \times r}
\end{array}\right], } \\
W_{w}= & {\left[\begin{array}{lll}
0_{r \times(n+r)} & 0_{r \times(n+r)} & I_{r}
\end{array}\right], } \\
W_{p h}= & {\left[\begin{array}{lll}
0_{n+r} & I_{n+r} & 0_{(n+r) \times r}
\end{array}\right] }
\end{aligned}
$$

Matrix $P_{1}$ has the structure of:

$$
P_{1}=\left[\begin{array}{cc}
P_{11} & 0 \\
P_{13} & P_{12}
\end{array}\right]
$$

where matrix $P_{1}$ satisfies $E^{T} P_{1}=P_{1} E$. Matrix $P_{11}>0$, $P_{12}$ is invertible and $P_{13}$ is a slack matrix.

$\tilde{K}$ can be computed by:

$$
\tilde{K}=\left(P_{1}\right)^{-1} X
$$

Then, the observer and controller gains can be obtained.

Proof: Let us consider the weighted constraint given by

$$
\int_{0}^{t} e^{T}(\sigma) Q e(\sigma) d \sigma \leq \gamma^{2} \int_{0}^{t} w^{T}(\sigma) w(\sigma) d \sigma
$$

where $\gamma$ is the attenuation level from the perturbation term to the error, and $Q$ is a known semi-positive-definite weighting matrix. It is well known that constraint (13) is satisfied if there exists a Lyapunov candidate function such that:

$$
\dot{V}(t)+e^{T}(t) Q e(t)-\gamma^{2} w^{T}(t) w(t)<0
$$

Choose a Lyapunov-Krasovskii functional candidate $V(t)=V_{1}(t)+V_{2}(t)+V_{3}(t)$, and $V_{1}(t), V_{2}(t), \quad V_{3}(t)$ are defined as:

$$
\begin{aligned}
& V_{1}(t)=e^{T}(t) E^{T} P_{1} e(t) \\
& V_{2}(t)=\int_{t-h}^{t} e^{T}(\omega) P_{2} e(\omega) d \omega \\
& V_{3}(t)=\int_{-h}^{0} \int_{t+v}^{t} e^{T}(\omega) E^{T} P_{3} E e(\omega) d \omega d v
\end{aligned}
$$

where matrix $P_{1}$ satisfies $E^{T} P_{1}=P_{1} E$.

The time derivatives of $V(t)$ is given by:

$$
\begin{aligned}
\dot{V}(t)= & 2 e^{T}(t) P_{1} E \dot{e}(t)+h \dot{e}^{T}(t) E^{T} P_{3} E \dot{e}(t)+e^{T}(t) P_{2} e(t) \\
& -e^{T}(t-h) P_{2} e(t-h)-\int_{t-h}^{t} \dot{e}^{T}(\omega) E^{T} P_{3} E \dot{e}(\omega) d \omega
\end{aligned}
$$

According to the Leibniz-Newton formula, the following equation is true:

$$
\Pi=\xi^{T}(t) Y E\left[e(t)-e(t-h)-\int_{t-h}^{t} \dot{e}(\omega) d \omega\right]=0
$$

where $Y$ is an appropriate dimensioned matrix, and $\xi(t)=\left[\begin{array}{lll}e^{T}(t) & e^{T}(t-h) & w^{T}(t)\end{array}\right]^{T}$. 
Adding $2 \Pi, \quad h \xi^{T}(t) Y P_{3}^{-1} Y^{T} \xi(t)$ and subtracting $\int_{t-h}^{t} \xi^{T}(t) Y P_{3}^{-1} Y^{T} \xi(t) d \omega$ in (16) give the following:

$$
\begin{aligned}
\dot{V}(t)= & 2 e^{T}(t) P_{1} E \dot{e}(t)+h \dot{e}^{T}(t) E^{T} P_{3} E \dot{e}(t) \\
& -\int_{t-h}^{t} \dot{e}^{T}(\omega) E^{T} P_{3} E \dot{e}(\omega) d \omega+e^{T}(t) P_{2} e(t) \\
& -e^{T}(t-h) P_{2} e(t-h)+h \xi^{T}(t) Y P_{3}^{-1} Y^{T} \xi(t) \\
& -\int_{t-h}^{t} \xi^{T}(t) Y P_{3}^{-1} Y^{T} \xi(t) d \omega \\
& +2 \xi^{T}(t) Y E\left[e(t)-e(t-h)-\int_{t-h}^{t} \dot{e}(\omega) d \omega\right] \\
= & \Omega+h \xi^{T}(t) Y P_{3}^{-1} Y^{T} \xi(t)+h \dot{e}^{T}(t) E^{T} P_{3} E \dot{e}(t) \\
& -\int_{t-h}^{t}\left[\xi^{T}(t) Y+\dot{e}^{T}(\omega) P_{3}\right] P_{3}^{-1}\left[Y^{T} \xi(t)+P_{3} \dot{e}(\omega)\right] d \omega
\end{aligned}
$$

Then, we know $\Omega+h \xi^{T}(t) Y P_{3}^{-1} Y^{T} \xi(t)+h \dot{e}^{T}(t) E^{T}$ $P_{3} E \dot{e}(t)<0$ which guarantees that $\dot{V}(t)$ is non-positive for non-zero $\xi(t)$. We can apply the Schur complement in it which yields the following:

$$
\left[\begin{array}{ccc}
\Omega & Y & \Phi \\
* & -P_{3} / h & 0 \\
* & * & -P_{3}^{-1} h^{-1}
\end{array}\right] \leq 0
$$

where $\Phi=\left[\begin{array}{lll}A_{0}-\tilde{K} \tilde{C} & A_{d} & R\end{array}\right]^{T}$. Pre- and Post-multiplying both sides of (17) with $\operatorname{diag}\left(I, I, P_{1}\right)$ and its transpose, we have (18).

$$
\left[\begin{array}{ccc}
\Omega & Y & \Psi \\
* & -P_{3} / h & 0 \\
* & * & -P_{1} P_{1} / P_{3} h
\end{array}\right] \leq 0
$$

where $\Psi=\Phi P_{1}$.

Define a variable $M$ such that $M \leq P_{1} P_{1} / P_{3}$ [21], and to satisfy this inequality, by using the Schur complement we have:

$$
\left[\begin{array}{ll}
M & P_{1} \\
P_{1} & P_{3}
\end{array}\right] \leq 0
$$

Then, we know that if (11) are satisfied, Theorem 1 follows.

\section{Fault-Tolerant Control with the Effect of Distur- bances}

If there are disturbances and actuator faults simultaneous in system (1), one can represent system (1) by

$$
\begin{aligned}
& \dot{x}(t)=A x(t)+B u(t)+F f(t)+D d(t) \\
& y(t)=C x(t)
\end{aligned}
$$

where $d(t) \in \mathrm{R}^{\mathrm{d}}$ is the unknown disturbance vector and $D$ is a known constant matrix.

Similar to (5), the state estimation error dynamic can be shown as:

$$
\begin{aligned}
\dot{e}_{x}(t)= & N e_{x}(t)+\left(H_{1} A-N H_{1}-L_{1} C\right) x(t) \\
& +\left(H_{1} B-G\right) u(t)+T e_{f}(t)+\left(T-H_{1} F\right) f(t) \\
& +H_{1} D d(t)
\end{aligned}
$$

Then, to provide robustness to disturbances and sensitivity to faults, the following equation must be satisfied:

$$
\begin{aligned}
& H_{1} A-N H_{1}-L_{1} C=0 \\
& G=H_{1} B \\
& T=H_{1} F \\
& H_{1} D=0
\end{aligned}
$$

As such, the dynamics of the state estimation error and the fault estimation error can be described by:

$$
\begin{aligned}
& \dot{e}_{x}(t)=N e(t)+T e_{f}(t) \\
& e_{f}(t)=e_{f}(t-h)-K_{1} C e_{x}(t)+k_{f}
\end{aligned}
$$

where $k_{f}$ is a known bound of the variation defined in (9).

To design the FTC law, we have the following assumption [22].

Assumption 3. $\operatorname{rank}(B, F)=\operatorname{rank}(B)$.

From Assumption 3, it is easy to know that the vector space spanned by the column of $F$ is a subset of the space spanned by the column vectors of $B$, that is $\operatorname{span}(F) \subseteq \operatorname{span}(B)$, which is equivalent to the existence of $B^{*}$ such that:

$$
\left(I-B B^{*}\right) F=0
$$

So, once a fault occurs, based on the accurate estimation of the fault, the following observer-based fault tolerant controller is activated to compensate the fault effect:

$$
u_{c}(t)=e_{u}(t)-B^{*} F \hat{f}(t)+r(t)
$$

where $r(t) \in \mathrm{R}^{\mathrm{m}}$ is the reference input.

As the system state $x(t)$ is unavailable, the system state estimation $\hat{x}(t)$ is substituted for $x(t)$, therefore, the controller gain error is given by:

$$
e_{u}(t)=K_{2} \hat{x}(t)=K_{2} x(t)-K_{2} e_{x}(t)
$$

where $K_{2} \in \mathrm{R}^{\mathrm{m} \times \mathrm{n}}$ is the feedback gain matrix.

If we assume that $r(t)=0$, by substituting (24) to (20), we can obtain

$$
\dot{x}(t)=A x(t)+B e_{u}(t)+F e_{f}(t)+D d(t)
$$


To organize the above equations into the form of descriptor systems, we introduce the "virtual dynamic" in $e_{f}(t)$ and $e_{u}(t)$. Then, the dynamics of these latter can be rewritten as:

$$
\begin{aligned}
& 0 \dot{e}_{u}(t)=-e_{u}(t)+K_{2} x(t)-K_{2} e_{x}(t) \\
& 0 \dot{e}_{f}(t)=-e_{f}(t)+e_{f}(t-h)-K_{1} C e_{x}(t)+k_{f}
\end{aligned}
$$

The combination of (21), (26) and (27) yields a descriptor system expressed as follows:

$$
E \dot{e}(t)=\left(A_{0}-\tilde{K} \tilde{C}_{1}-\tilde{H} \tilde{C}_{2}\right) e(t)+A_{d} e(t-h)+R w(t)
$$

where $\quad E=\operatorname{diag}\left\{I_{n}, I_{n}, 0_{r}, 0_{m}\right\}, \quad e(t)=\left[e_{x}^{T}(t) \quad x^{T}(t)\right.$ $\left.e_{f}^{T}(t) \quad e_{u}^{T}(t)\right]^{T}, w(t)=\left[\begin{array}{ll}d^{T}(t) & k_{f}^{T}\end{array}\right]^{T}$,

$A_{d}=\operatorname{diag}\{0,0, I, 0\}$

$$
\begin{gathered}
R=\left[\begin{array}{ll}
0 & 0 \\
D & 0 \\
0 & I \\
0 & 0
\end{array}\right], A_{0}=\left[\begin{array}{cccc}
H_{1} A & 0 & T & 0 \\
0 & A & F & B \\
0 & 0 & -I & 0 \\
0 & 0 & 0 & -I
\end{array}\right], \tilde{K}=\left[\begin{array}{c}
K \\
0 \\
K_{1} \\
0
\end{array}\right], \\
\tilde{C}_{1}=\left[\begin{array}{c}
C^{T} \\
0 \\
0 \\
0
\end{array}\right]^{T}, \tilde{C}_{2}=\left[\begin{array}{c}
I \\
-I \\
0 \\
0
\end{array}\right]^{T}, \tilde{H}=\left[\begin{array}{c}
0 \\
0 \\
0 \\
K_{2}
\end{array}\right]^{T}
\end{gathered}
$$

Theorem 2. System (28) describing the state estimation error $e_{x}(t)$, the fault estimation error $e_{f}(t)$, the system state $x(t)$ and the controller gain error $e_{u}(t)$ is stable, and the gain from the perturbation-like term $w(t)$ to the different errors is bounded by $\gamma>0$ if there exist some matrices $P_{1}, P_{2}>0, P_{3}>0, \tilde{M}>0$, matrix $Y$ with appropriate dimensions such that the following matrix inequalities hold:

$$
\begin{aligned}
& {\left[\begin{array}{ccc}
\tilde{\Omega} & Y & \tilde{\Psi} \\
* & -P_{3} / h & 0 \\
* & * & -\tilde{M} / h
\end{array}\right] \leq 0} \\
& {\left[\begin{array}{cc}
\tilde{M} & P_{1} \\
P_{1} & P_{3}
\end{array}\right] \leq 0}
\end{aligned}
$$

where $\tilde{\Omega}, \tilde{\Psi}$ are shown as follows:

$$
\begin{aligned}
& \tilde{\Omega}=W_{p}^{T} Q W_{p}-\gamma^{2} W_{w}^{T} W_{w}+W_{p}^{T} P_{2} W_{p}-W_{p h}^{T} P_{2} W_{p h} \\
& +\operatorname{sym}\left(W_{p}^{T}\left(P_{1} A_{0}-X_{1} \tilde{C}_{1}-X_{2} \tilde{C}_{2}\right) W_{p}\right. \\
& +W_{p}^{T} P_{1} A_{d} W_{p h}+W_{p}^{T} P_{1} R W_{w} \\
& \left.+Y E W_{p}-Y E W_{p h}\right) \\
& \tilde{\Psi}=\left[\left(P_{1} A_{0}-X_{1} \tilde{C}_{1}-X_{2} \tilde{C}_{2}\right) P_{1} A_{d} P_{1} R\right]^{T} \\
& X_{1}=\left[\begin{array}{llll}
X_{11}^{T} & 0 & X_{13}^{T} & 0
\end{array}\right], \quad X_{2}=\left[\begin{array}{llll}
0 & 0 & 0 & X_{24}^{T}
\end{array}\right] \\
& X_{1}=P_{11} K, \quad X_{13}=P_{13} K_{1}, \quad X_{24}=P_{14} K_{2} \\
& W_{p}=\left[\begin{array}{lll}
I_{n+n+m+r} & 0_{n+n+m+r} & 0_{(n+n+m+r) \times(d+r)}
\end{array}\right] \text {, } \\
& W_{w}=\left[\begin{array}{lll}
0_{(d+r) \times(n+n+m+r)} & 0_{(d+r) \times(n+n+m+r)} & I_{d+r}
\end{array}\right], \\
& W_{p h}=\left[\begin{array}{lll}
0_{n+n+m+r} & I_{n+n+m+r} & 0_{(n+n+m+r) \times(d+r)}
\end{array}\right]
\end{aligned}
$$

and $P_{1}$ have the structure of the form:

$$
P_{1}=\left[\begin{array}{cccc}
P_{11} & 0 & 0 & 0 \\
0 & P_{12} & 0 & 0 \\
0 & P_{15} & P_{13} & 0 \\
0 & P_{16} & 0 & P_{14}
\end{array}\right]
$$

where $P_{11}>0, P_{12}>0$ are positive-definite matrices, matrices $P_{13}, P_{14}$ are invertible and matrices $P_{15}, P_{16}$ are the slack matrices with appropriate dimensions.

The observer and controller gains are then computed by:

$$
K=P_{11}^{-1} X_{11}, \quad K_{1}=P_{13}^{-1} X_{13}, K_{2}=P_{14}^{-1} X_{24}
$$

Following the similar steps with the previous proof of Theorem 1, the sufficient LMI conditions proposed in Theorem 2 follow.

Remark 3. Compared with the references of Jia [13] and HAMDI [23], by which our paper is mainly motivated, our method brings some novelties which can be summarized as follows. (1) The system under the study is different. Jia and HAMDI consider LPV models, and in our study the linear system is investigated. However, the results of linear systems can be applied to LPV models. (2) The main issues researched are different. Jia and HAMDI only consider the problem of fault estimation. Our method can deal with the fault estimate and FTC together. (3) Our method can deal with the uncertainties and disturbances simultaneously because we use the UIO to estimate system states, the 
advantages of which is that we can group the uncertainties and disturbances together [24] and estimate system states accurately. (4) Most importantly, the way we deal with the fault update law is different. We refer the fault iterative update law to time-delay control, which makes the obtained results new. The reason is that we can adopt the Lyapunov-Krasovskii theorem other than the Lyapunov-Razumikhin approach to prove the stability, and the results of using the Lyapunov-Krasovskii approach are usually less conservative than those using the Lyapunov-Razumikhin approach [20].

\section{Numerical Example}

In this section, we will provide a numerical example to demonstrate the effectiveness of the design method proposed in the previous section. This example is taken from [23] and defined by:

$$
\begin{aligned}
A & =\left[\begin{array}{cccc}
-1.85 & 1 & 0 & 0 \\
-1 & -1.05 & 0 & 0 \\
-1.8 & -1 & -0.8 & 0 \\
-1 & 0 & 0 & -1.1
\end{array}\right], F=\left[\begin{array}{c}
0 \\
0.55 \\
0 \\
1
\end{array}\right], \\
B & =\left[\begin{array}{cc}
0.95 & 1 \\
1 & 0.4 \\
1 & 0 \\
-0.1 & 0
\end{array}\right], C=\left[\begin{array}{llll}
1 & 0 & 1 & 0 \\
0 & 1 & 0 & 1 \\
0 & 0 & 1 & 1
\end{array}\right]
\end{aligned}
$$

First, we would consider the problem of fault estimation.

We assume that the fault $f(t)$ is a sinusoidal signal applied for $30 \mathrm{~s} \leq t \leq 60 \mathrm{~s}$. The initial values of the original states and the estimated states are all equal to zero, the signal of fault is $\sin (t)$, the nominal input signal $r(t)=0$.

Following the design steps of UIO, we have:

$$
\begin{aligned}
& H_{1}=\left[\begin{array}{cccc}
0.6190 & -0.0476 & -0.2381 & 0.0952 \\
-0.0476 & 0.6190 & 0.0952 & -0.2381 \\
-0.2381 & 0.0952 & 0.4762 & -0.1905 \\
0.0952 & -0.2381 & -0.1905 & 0.4762
\end{array}\right], \\
& H_{2}=\left[\begin{array}{cccc}
0.3810 & 0.0476 & -0.1429 \\
0.0476 & 0.3810 & -0.1429 \\
0.2381 & -0.0952 & 0.2857 \\
-0.0952 & 0.2381 & 0.2857
\end{array}\right]
\end{aligned}
$$

If we choose $Q=I_{n+r}, \gamma^{2}=0.01$ and by using the LMI toolbox to determine the gain and the observer matrices, these inequalities are fulfilled with:

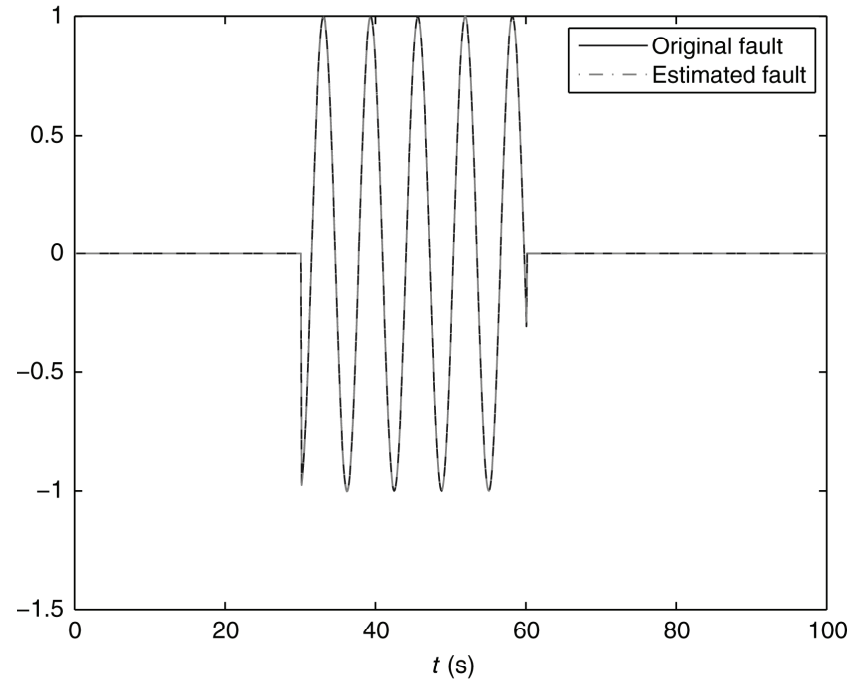

Figure 1. Fault and its estimation.

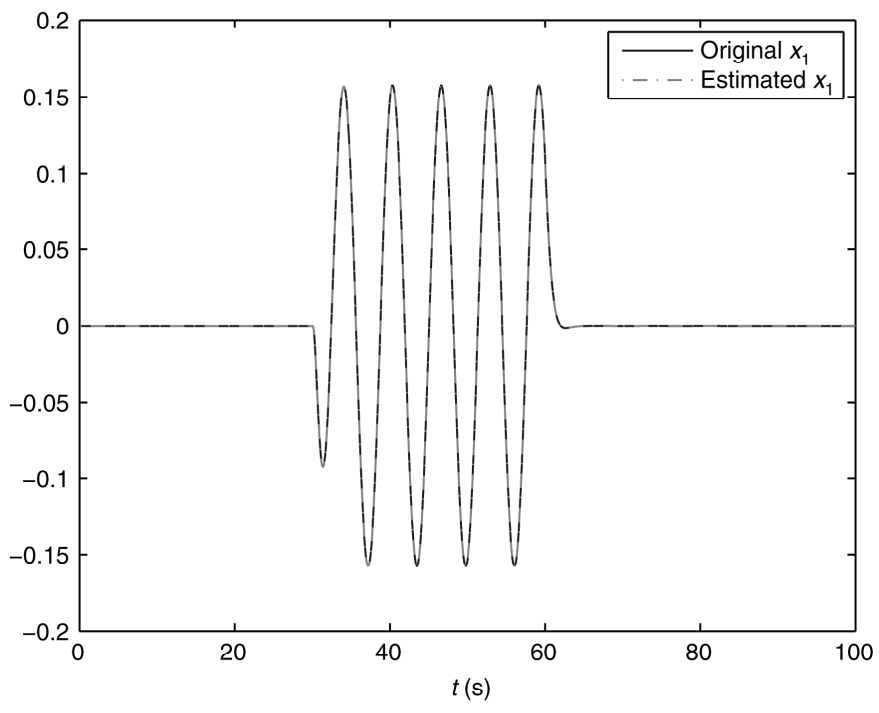

Figure 2. Comparison of state $x_{1}$ and observer state $\hat{x}_{1}$.

$$
\begin{aligned}
K & =\left[\begin{array}{ccc}
-0.8608 & -0.9439 & -1.1484 \\
-0.2645 & 0.5497 & -0.4736 \\
0.9277 & -0.3870 & 0.9003 \\
-0.1971 & 0.7085 & 0.6799
\end{array}\right], \\
K_{1} & =\left[\begin{array}{lll}
-1.1173 & 0.4418 & 0.1977
\end{array}\right] \times 10^{4}
\end{aligned}
$$

The state estimation and unknown values of the estimation given by the ILO are shown in Figs. 1-5.

The simulation results are shown in Figs. 1-5. In Fig. 1, the real fault and its estimate are depicted. Figures 2-5 compare the state variables of the system with state estimation variables of UIO. From these figures, one can observe that the fault $f(t)$ appears between $t=30 \mathrm{~s}$ and $t=60 \mathrm{~s}$. Clearly, when the fault occurs, the system states 


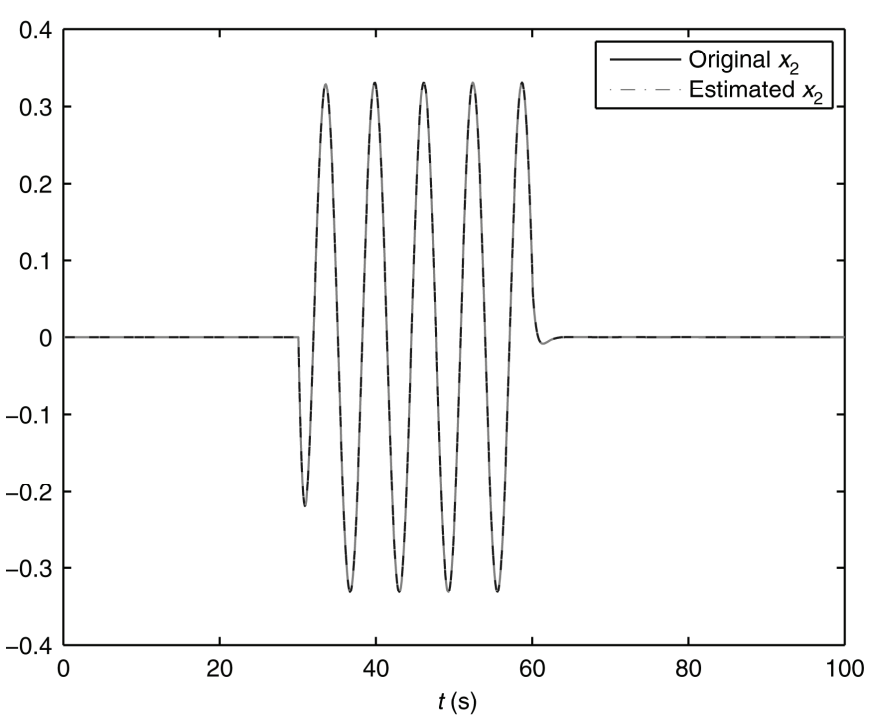

Figure 3. Comparison of state $x_{2}$ and observer state $\hat{x}_{2}$.

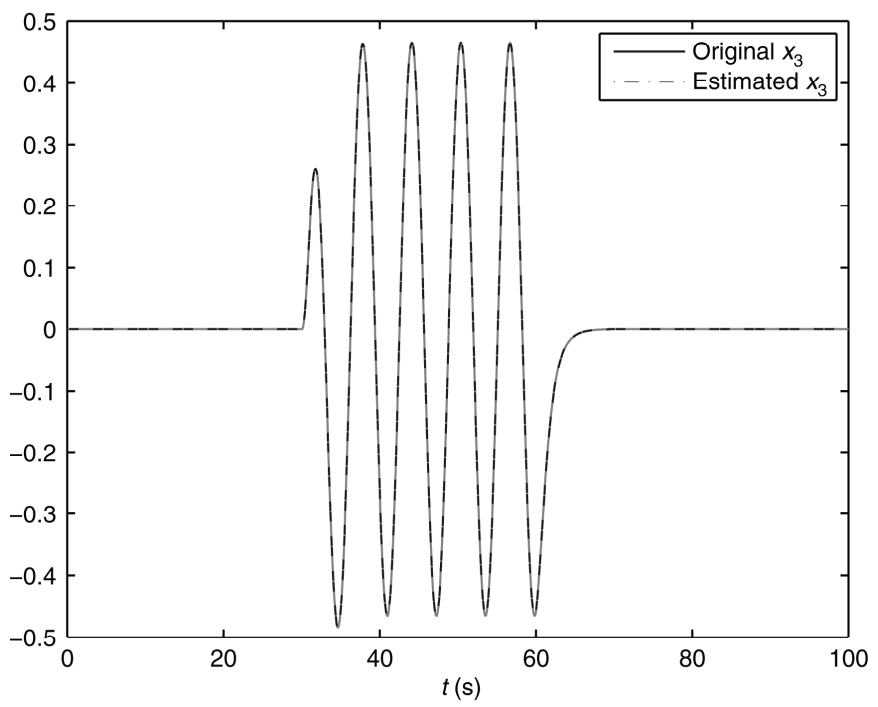

Figure 4. Comparison of state $x_{3}$ and observer state $\hat{x}_{3}$.

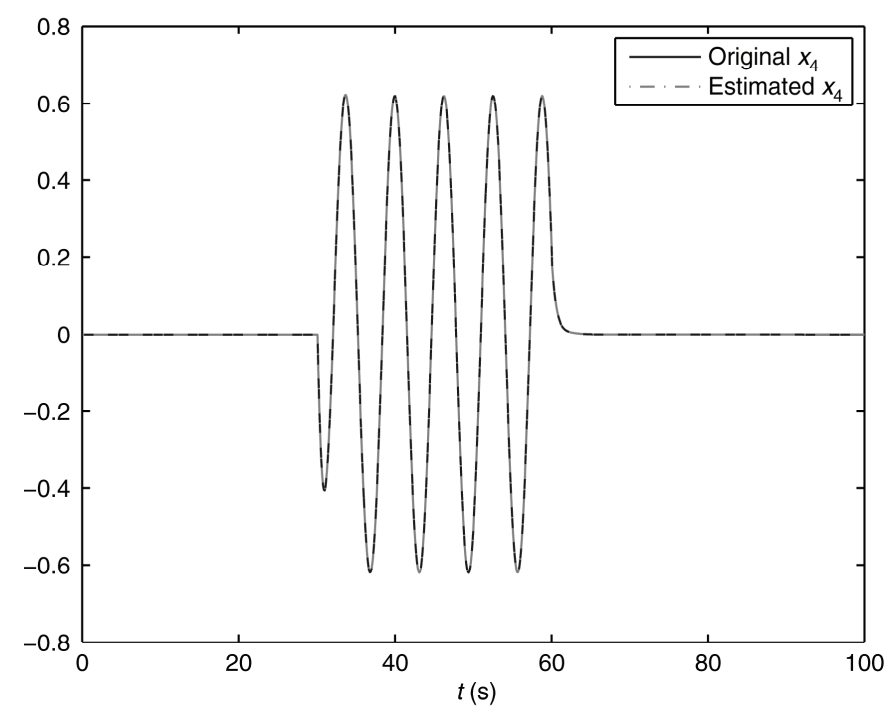

Figure 5. Comparison of state $x_{4}$ and observer state $\hat{x}_{4}$. deviate from their original trajectories because of the effects of the fault. But the fault and the system states can still be estimated correctly which illustrate that the observer is effective.

In the following, we will consider the problems of fault estimation and FTC with effects of disturbances.

To show the effectiveness, we consider two different faults which occur in different pathways. An incipient fault $f(t)=0.001 t$ and a time-varying fault $f(t)=\sin (0.5 t) \cos (t-6)$ affect the system behaviour at $30 \mathrm{~s} \leq t \leq 60 \mathrm{~s}$.

When choosing the parameter of the sampling time interval $h=0.01$, letting the nominal input signal $r(t)=\left[\begin{array}{ll}0.1 & -0.1\end{array}\right]^{T}$, and following the design steps of UIO, we have:

$$
\begin{aligned}
& H_{1}=\left[\begin{array}{cccc}
0.4314 & -0.3455 & -0.4644 & -0.2414 \\
-0.0626 & 0.5953 & 0.0772 & -0.2649 \\
-0.1355 & 0.2581 & 0.5999 & -0.0065 \\
-0.0249 & -0.4288 & -0.3353 & 0.2607
\end{array}\right], \\
& H_{2}=\left[\begin{array}{ccc}
0.5686 & 0.3455 & -0.1042 \\
0.0626 & 0.4047 & -0.1398 \\
0.1355 & -0.2581 & 0.2646 \\
0.0249 & 0.4288 & 0.3105
\end{array}\right]
\end{aligned}
$$

If we choose $Q=I_{n+r}, \gamma^{2}=1.982812$, by using the LMI toolbox to determine the gain and the observer matrices, these inequalities are fulfilled with:

$$
\begin{gathered}
K=\left[\begin{array}{ccc}
1.7515 & 1.4213 & -1.2156 \\
0.4781 & 0.8172 & -0.4161 \\
0.1184 & -1.5244 & 1.6036 \\
0.0187 & 1.6171 & 0.3016
\end{array}\right], \\
K_{2}=\left[\begin{array}{cccc}
-0.3770 & -0.3068 & -0.0631 & 0.3115 \\
-0.0553 & 0.1115 & 0.0661 & 0.2670
\end{array}\right] \\
K_{1}=\left[\begin{array}{cccc}
2.2968 & -1.6988 & -0.2936 \\
2.0187 & 0.8458 & -2.2639
\end{array}\right] \times 10^{8}
\end{gathered}
$$

The simulation results are shown in Figs. 6-12. In Figs. 6 and 7, the real faults and their estimates are depicted; Fig. 12 shows the system input signal which include the control input author applied and FTC feedback control signal.

Figures 8-11 describe the response curves of $\hat{x}_{i}$ $(i=1,2,3,4)$ in case of fault free and faulty with and without FTC. From these figures, we can find that the curves of $\hat{x}_{i}$ in case of faulty with FTC are not affected by the fault 


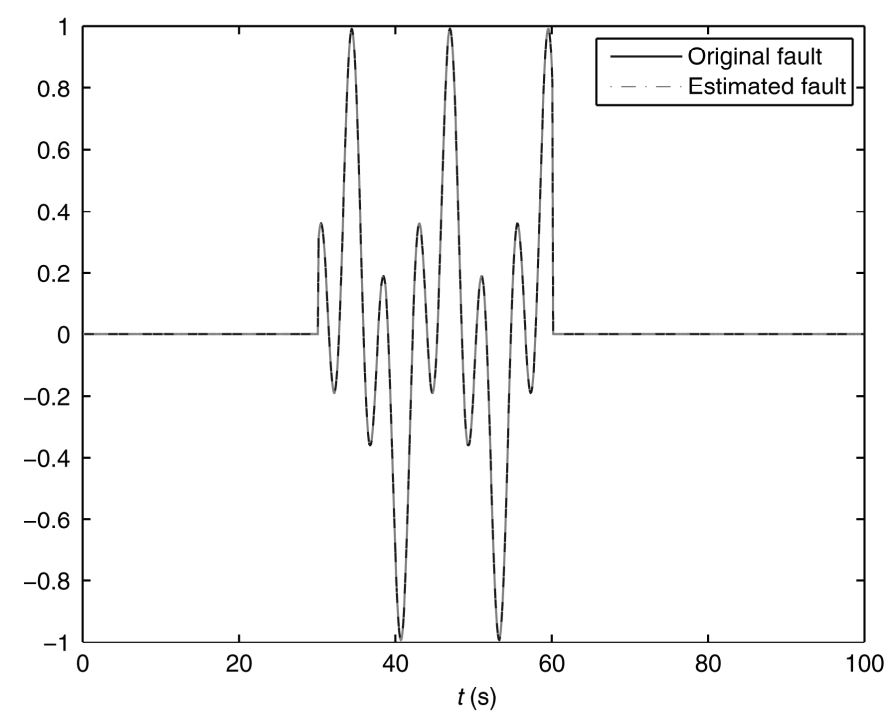

Figure 6. Time-varying fault and its estimation.

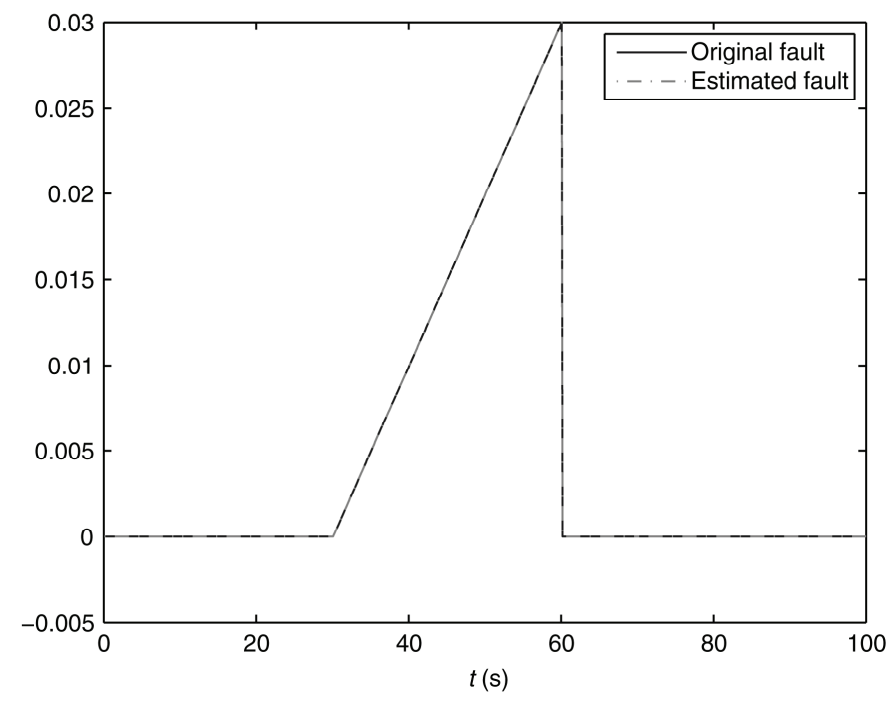

Figure 7. Incipient fault and its estimation.

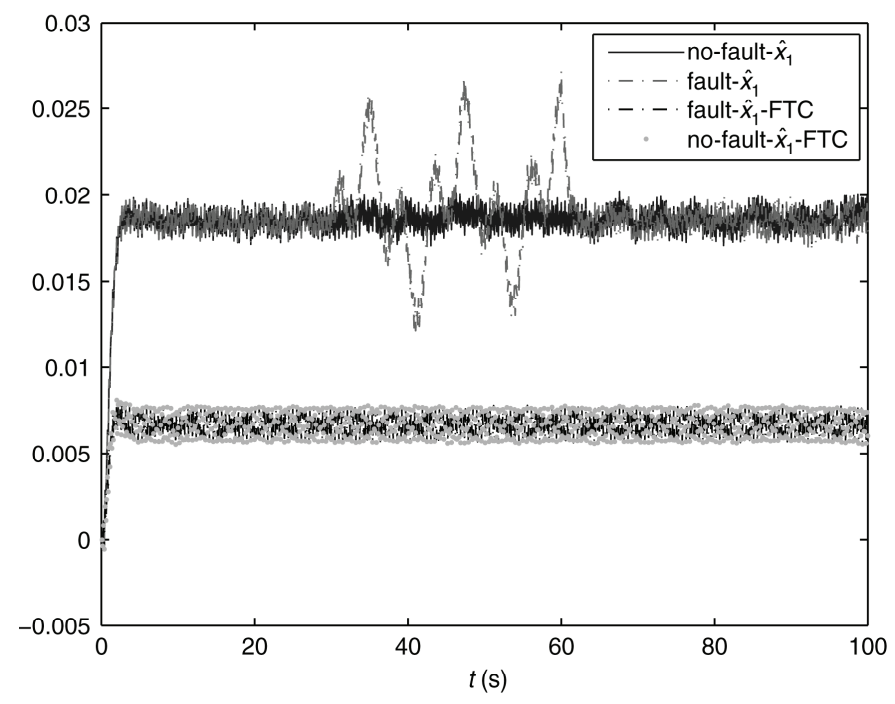

Figure 8. Comparison of state $\hat{x}_{1}$.

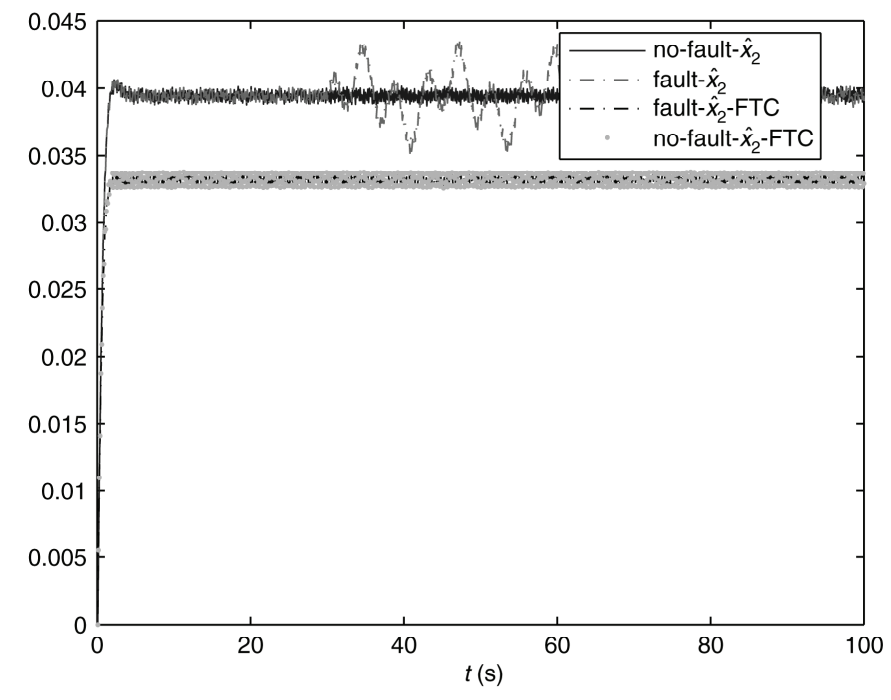

Figure 9. Comparison of state $\hat{x}_{2}$.

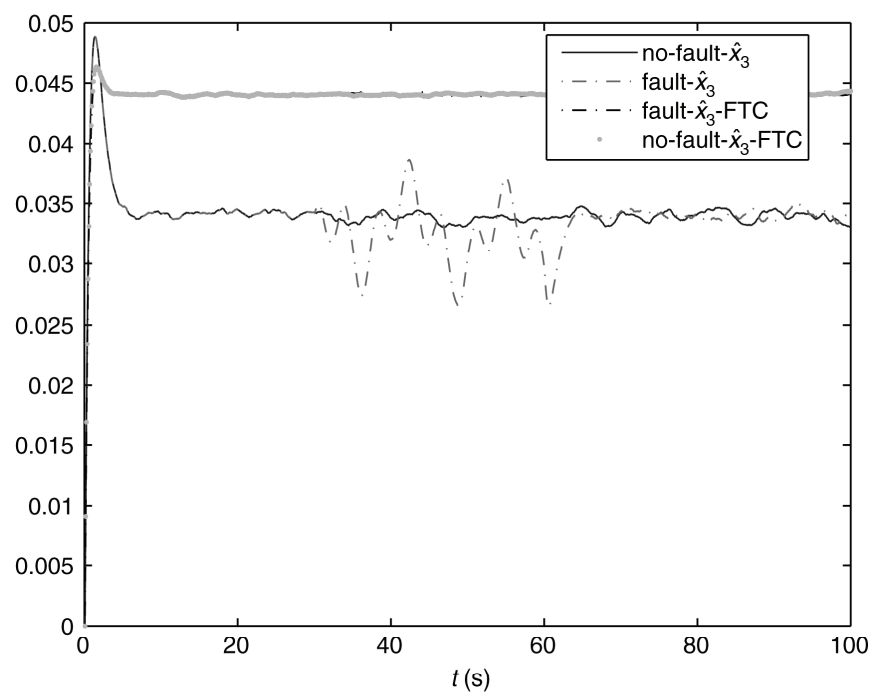

Figure 10. Comparison of state $\hat{x}_{3}$.

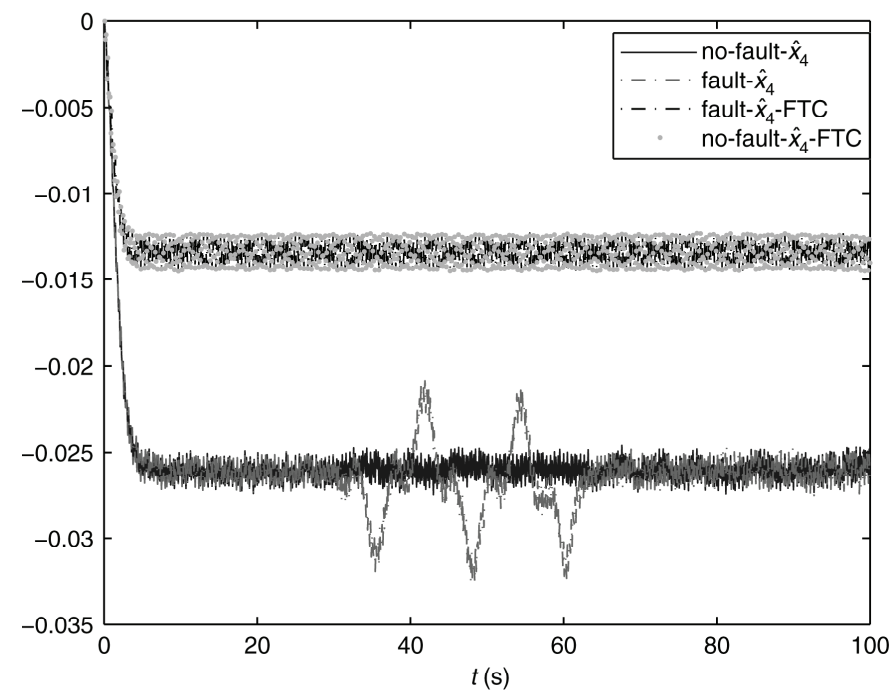

Figure 11. Comparison of state $\hat{x}_{4}$. 


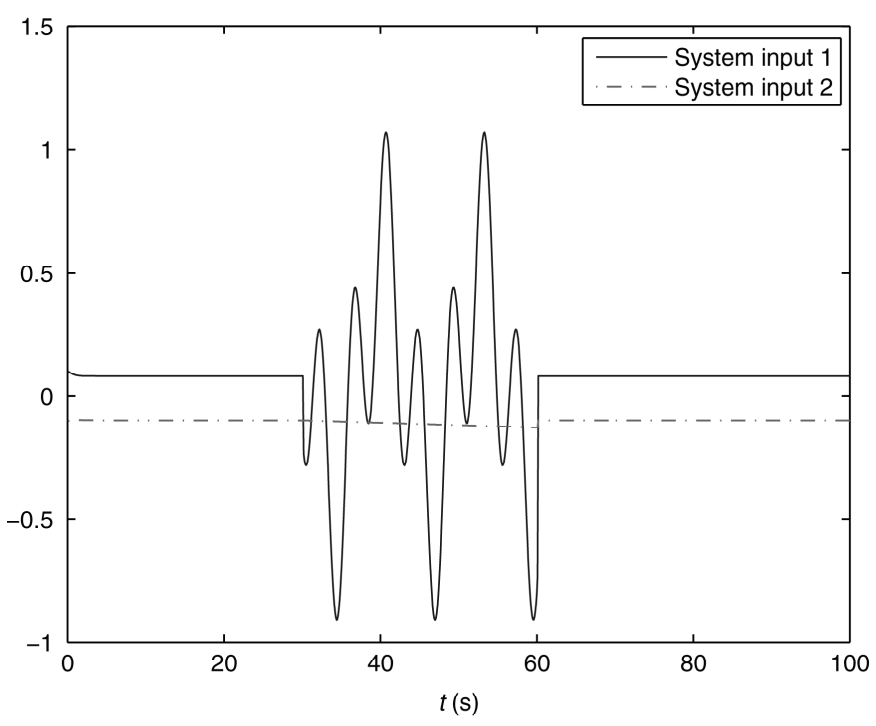

Figure 12. Control input signal $u(t)$.

because the FTC eliminates these effects. On the other hand, the curves of $\hat{x}_{i}$ without FTC deviate from their trajectory. Besides that, it is considerable to note that the curves with FTC and those without FTC follow different trajectories, this is because the FTC controller not only include the term of the estimated fault to eliminate effects of the real fault but also the term of feedback control $K_{2} \hat{x}_{i}$ which is the cause of the difference between the curves with and without FTC. All these figures illustrate that the observer and the FTC are effective.

\section{Conclusion}

In this paper, a fault estimation and FTC approach has been provided based on iterative learning and UIO for linear systems with actuator faults. Compared with some existing results, the novelty of this paper is that our approach takes the iterative learning update law as time-delay control, which is used to estimate the unknown input. This iterative learning update law combined with the input gain error, the system state and the state estimation error yields a kind of time-delay descriptor system, and moreover, the stability of this time-delay descriptor system can be proved by using the Lyapunov-Krasovskii stability theory. Finally, a numerical example has been given to illustrate the effectiveness of the fault estimation and FTC method.

\section{Acknowledgements}

The authors would like to thank the reviewers and editors for their useful comments and suggestions.

\section{References}

[1] Y. Xiong and M. Saif, Robust fault detection and isolation via a diagnostic observer, International Journal of Robust and Nonlinear Control, 10, 2000, 1175-1192.

[2] C.W. de Silva and K. Wong, Online fault identification and fault tolerant control of a multi-module manipulator, International Journal of Robotics and Automation, 25(3), 2010, 217-228.
[3] C. Keliris, M.M. Polycarpou, and T. Parisini, A robust nonlinear observer-based approach for distributed fault detection of input-output interconnected systems, Automatica, 53, 2015, 408-415.

[4] D. Zhu, J. Liu, and S.X. Yang, Particle swarm optimization approach to thruster fault-tolerant control of unmanned underwater vehicles, International Journal of Robotics and Automation, 26(3), 2011, 282-287.

[5] S. Rahme and N. Meskin, Adaptive sliding mode observer for sensor fault diagnosis of an industrial gas turbine, Control Engineering Practice, 38, 2015, 57-74.

[6] H. Hamdi, M. Rodrigues, C. Mechmeche, et al., State estimation for polytopic LPV descriptor systems: Application to fault diagnosis, Proc. 7th IFAC Symp. Fault Detection, Supervision and Safety of Technical Processes, Barcelona, Spain, 2009, 438-443.

[7] B. Jiang, K. Zhang, and P. Shi, Less conservative criteria for fault accommodation of time-varying delay systems using adaptive fault diagnosis observer, International Journal of Adaptive Control and Signal Processing, 24(4), 2010, 322-334.

[8] D. Du, B. Jiang, and P. Shi, Active fault-tolerant control for switched systems with time delay, International Journal of Adaptive Control and Signal Processing, 25, 2011, 466-480.

[9] W. Chen and M. Saif, Observer-based strategies for actuator fault detection, isolation and estimation for certain class of uncertain nonlinear systems, IET Control Theory and Applications, 1(6), 2007, 672-1680.

[10] X.J. Li and G.H. Yang, Dynamic observer-based robust control and fault detection for linear systems, IET Control Theory and Applications, 6(17), 2013, 2657-2666.

[11] X. Wang and Y. Pi, Trajectory tracking control of a hydraulic parallel robot manipulator with lumped disturbance observer, International Journal of Robotics and Automation, 28(2), 2013, 103-111.

[12] W. Chen and M. Joo Er, Simultaneous identification of timevarying parameters and estimation of system states using iterative learning observer, IEEE Transactions on Automatic Control, 49(10), 2004, 1869-1875.

[13] Q.X. Jia, W. Chen, Y.C. Zhang, et al., Robust fault reconstruction via learning observers in linear parameter-varying systems subject to loss of actuator effectiveness, IET Control Theory and Applications, 8(1), 2014, 42-50.

[14] L.N. Yao, V. Cocquempot, and D.S. Wang, Fault tolerant control for a class of nonlinear systems using an iterative learning observer, 2nd Int. Asia Conf. Informatics in Control Automation and Robotics, WuHan, China, 2010, 373-376.

[15] W. Chen and M. Saif, An iterative learning observer-based approach to fault detection and accommodation in nonlinear systems, Proc. 40th lEEE Conf. Decision and Control, Orlando, USA, 2001, 4469-4474.

[16] T. Bouarar, B. Marx, and D. Maquin, Fault tolerant control design for uncertain Takagi-Sugeno systems by trajectory tracking: A descriptor approach, IET Control Theory and Applications, 7(14), 2013, 1793-1805.

[17] K. Youcef-Toumi and S.T. Wu, Input/output linearization using time delay control, American Control Conference, Boston, USA, 1991, 10-19.

[18] X. Zhang, M.M. Polycarpou, and T. Parisini, Integrated design of fault diagnosis and accommodation schemes for a class of nonlinear systems, Proc. 40th IEEE Conf. Decision and Control, Orlando, Orlando, USA, 2001, 1448-1453.

[19] G.J. Zhang, C.S. Han, and L.G. Wu, Admissibility and $\mathrm{H} \infty$ performance analysis of T-S fuzzy descriptor systems with time-varying delay, The 2nd Int. Conf. Intelligent Control and Information Processing, Harbin, China, 2011, 71-81.

[20] Y. Zhao, J.L. Wu, and P. Shi, H $\infty$ control of nonlinear dynamic systems: A new fuzzy delay fractioning approach, IET Control Theory and Applications, 3(7), 2009, 917-928.

[21] C. Peng, D. Yue, and Y.C. Tian, New approach on robust delay-dependent $\mathrm{H} \infty$ control for uncertain $\mathrm{T}-\mathrm{S}$ fuzzy systems with interval time-varying delay, IEEE Transactions on Fuzzy Systems, 17(4), 2009, 890-900.

[22] K. Zhang, B. Jiang, and V. Cocquempot, Fast adaptive fault estimation and accommodation for nonlinear time-varying delay systems, Asian Journal of Control, 11(6), 2009, 643-652. 
[23] H. Hamdi, M. Rodrigues, C. Mechmeche, et al., Fault detection and isolation for linear parameter varying descriptor systems via proportional integral observer, International Journal of Adaptive Control and Signal Processing, 26, 2011, 2-16.

[24] M. Saif and Y Guan, A new approach to robust fault detection and identification, IEEE Transactions on Aerospace and Electronic Systems, 29(3), 1993, 685-695.

\section{Biographies}

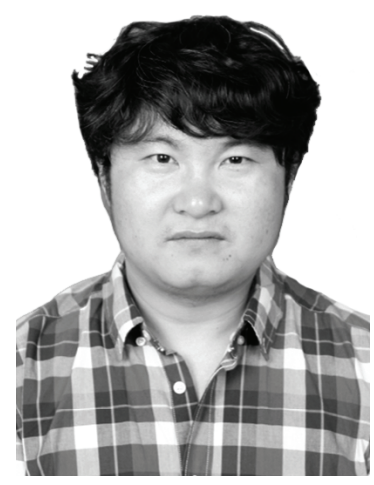

ShiLei Zhao received his B.E. and M.S. degree in automation from Harbin University of Science and technology, China, in 2001 and 2005, respectively. He received his Ph.D. degree in control science and engineering from Harbin Institute of Technology, Harbin, China, in 2010. He is currently an associate professor of school of software with Harbin University of Science and technology. His research interests include fault diagnosis and fault tolerant control.

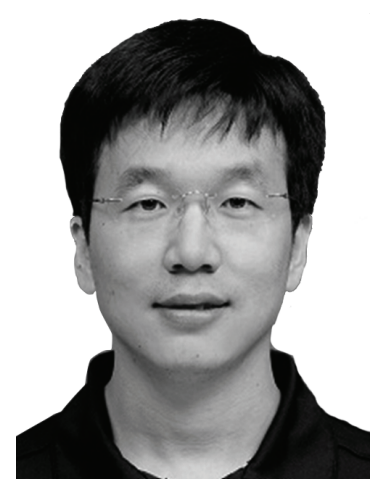

TieJun Zhang was born in Heilongjiang Province, China. He received his Bachelor, Master and Ph.D. degree of Computer Science from Harbin Institute of Technology, China. Now, he is an assistant professor in Harbin University of Science and Technology. His research interests include information security, signal processing, image processing and artificial intelligence.

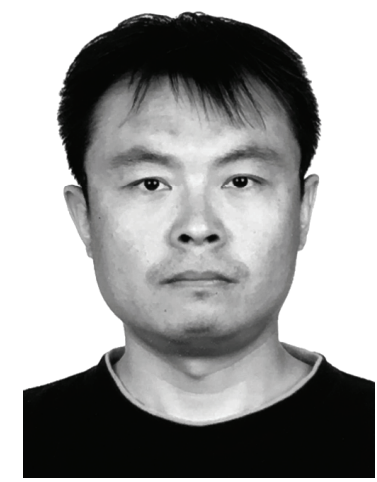

YuPeng Liu received his Bachelor and Master degrees from Harbin University of Science and technology, China, and the Ph.D. degree from Harbin Institute of Technology, Harbin, China. His research interests focus on natural language and artificial intelligence. 